\title{
Strength Testing in Motor Neuron Diseases
}

\author{
Jeremy M. Shefner ${ }^{1}$
}

Published online: 6 September 2016

(C) The American Society for Experimental NeuroTherapeutics, Inc. 2016

\begin{abstract}
Loss of muscle strength is a cardinal feature of all motor neuron diseases. Functional loss over time, including respiratory dysfunction, inability to ambulate, loss of ability to perform activities of daily living, and others are due, in large part, to decline in strength. Thus, the accurate measurement of limb muscle strength is essential in therapeutic trials to best understand the impact of therapy on vital function. While qualitative strength measurements show declines over time, the lack of reproducibility and linearity of measurement make qualitative techniques inadequate. A variety of quantitative measures have been developed; all have both positive attributes and limitations. However, with careful training and reliability testing, quantitative measures have proven to be reliable and sensitive indicators of both disease progression and the impact of experimental therapy. Quantitative strength measurements have demonstrated potentially important therapeutic effects in both amyotrophic lateral sclerosis and spinobulbar muscular atrophy, and have been shown feasible in children with spinal muscular atrophy. The spectrum of both qualitative and quantitative strength measurements are reviewed and their utility examined in this review.
\end{abstract}

Keywords Motor neuron disease - Spinal muscular atrophy · Manual muscle testing $\cdot$ TQNE $\cdot$ Hand held dynamometry

Jeremy M. Shefner

Jeremy.shefner@dignityhealth.org

1 Department of Neurology, Barrow Neurological Institute, Phoenix, AZ 85013, USA

\section{Introduction}

A clinical hallmark of motor neuron diseases is a progressive loss of strength. This loss underlies much of the disability that patients encounter, and is a major driver of healthcare costs associated with this constellation of diseases. Although other factors, such as upper motor neuron burden, may alter function, eating, breathing, speaking, ambulation, and fine motor control, all are dramatically affected by changes in muscle strength. A wide range of outcome measures have been employed in clinical trials, including survival, functional scales, and measures of specific functions such as vital capacity, sniff nasal inspiratory pressure, timed up and go test, walking distance for a defined period of time, and many others. However, as all of these are, in large part, a function of muscle strength, direct strength measurements have been a part of the vast majority of most clinical trials of experimental therapies for motor neuron disease. It is critical, therefore, that strength measurements be sensitive, repeatable, and performed in the same manner across study centers in multicenter clinical trials. This review will discuss the ways that muscle strength has been measured in clinical trials, and address the positive attributes and limitations of the various methods. The relationships between strength measures and other outcome measures will also be addressed.

\section{Methods of Strength Assessment}

\section{Manual Muscle Testing}

Manual muscle testing (MMT) was first described in 1912 to assess the status of patients with poliomyelitis [1]. In modern clinical settings, strength is most often assessed using the MMT scale established by the Medical Research Council of 
the Royal College of Physicians and Surgeons [2]. In its original form, this scale grades strength of individual muscles on a scale from 0 to 5 , with 0 representing no muscle function and 5 indicating normal strength. Grade 1 implies observation of muscle activation without movement, grade 2 requires the ability to move with gravity eliminated as a force, grade 3 means that a muscle can move a limb against gravity, and grade 4 requires good but not normal muscle power. In clinical trial settings, this scale has sometimes been expanded using either pluses or minuses or equivalently to 10 points with similar anchors but the ability to grade these subjective impressions in a somewhat finer manner.

MMT strength grading has been used in a number of clinical trial settings. In the phase III trial of riluzole versus placebo in patients with amyotrophic lateral sclerosis (ALS), a statistically significant benefit of riluzole was noted with respect to survival, with a suggestion of a dose-dependent trend toward increased efficacy with higher doses [3, 4]. However, MMT strength grading showed no difference from placebo, or any hint of a dose effect. In a later study comparing riluzole serum levels to both survival and MMT muscle testing, no effect of riluzole concentration was found on either measure [5]. In a phase III study of minocycline in ALS, a statistically significant trend toward faster progression in the ALS Functional Rating Scale-revised (ALSFRS-R) in patients treated with minocycline versus placebo; there was a trend in the same direction for MMT, but this was not significant [6]. A large, phase II trial of TCH346 in ALS showed a trend toward detriment on most outcome measures assessed but no effect on MMT [7]. Other studies have employed MMT testing in ALS, spinobulbar muscular atrophy, and spinal muscular atrophy (SMA) [8-12]. However, in most studies, no therapeutic benefit was noted with any outcome measure, so that the relative sensitivity of MMT testing compared with other measures could not be assessed. One study that suggested a therapeutic benefit in spinobulbar muscular atrophy has been recently reported; in this study, clenbuterol treatment was associated with a benefit as measured by the 6-min walk test, while no effect was noted in strength as measured with MMT testing [13].

To evaluate the properties of MMT testing as an outcome measure in ALS, the Great Lakes ALS Consortium performed a multicenter natural history study, evaluating patients with ALS longitudinally every 3 months for 1 year [14]. Eighteen muscle groups were evaluated bilaterally for a total of 36 muscles tested; evaluators all attended a training course to maximize consistency of measurement and technique. A 10point scale was used. Coefficient of variation of rate of change $[\mathrm{CoV}(\mathrm{r})]$ was calculated for single muscles, as well as averages from 2 to 36 muscle groups. $\mathrm{CoV}(\mathrm{r})$ is a measure that incorporates variability from reliability of measurement, as well as intrinsic variability of progression from patient to patient. Not surprisingly, $\mathrm{CoV}(\mathrm{r})$ was improved as more muscles were averaged together; with 36 muscle groups, $\mathrm{CoV}(\mathrm{r})$ was as good or better than many other ALS outcome measures, including the commonly used ALSFRS-R and vital capacity.

\section{Quantitative Muscle Testing}

Although the abovementioned studies show that MMT testing can be performed reliably in patients with motor neuron diseases, the measure itself is subjective, and it is clear that the scaling does not meet the requirements of an interval scale. The fact that several studies suggesting therapeutic change using other outcome measures did not show effects on MMT muscle strength testing also raises questions regarding the sensitivity of this measure with respect to its ability to detect meaningful therapeutic benefit. For these reasons, a method to measure muscle strength quantitatively is potentially attractive.

The relationship between MMT strength testing and isometric strength as measured by a strain gauge was originally evaluated by van der Ploeg et al. [15]. In this study, classic MMT strength grading of the biceps was compared with isometric strength (Fig. 1); antigravity strength (MMT grade 3) only required $2 \%$ of maximal biceps strength, with grades 4 and 5 spanning the remaining dynamic range of the muscle. It was noted that approximately $80 \%$ of the strength range of the biceps was graded as 4 . Thus, in a clinical trial, a patient could lose $>50 \%$ of his/her muscle power without a change in MMT level. Other studies have showed that a given level of isometric strength is variably graded by different trained evaluators, and by the same evaluator over repeated testing sessions. Andres et al. [16] showed that left ankle dorsiflexion of $40 \%$ of normal maximal force was graded between 3 and 8 by different evaluators using the modified 10-point MMT grading system. Given the fact that most of a muscle's dynamic range can fall within 1 MMT grade, and the fact that different evaluators grade the same strength markedly differently, a quantitative muscle measurement system is clearly to be desired.

A variety of quantitative measures have been employed, some using strain gauges and others with hand-held myometers [15, 17]. However, the first well-defined method to be used in clinical trials of motor neuron disease was developed by Munsat and Andres [16, 18-21]. Named the Tufts Quantitative Neuromuscular Evaluation (TQNE), the entire instrument included measurements of muscle strength,

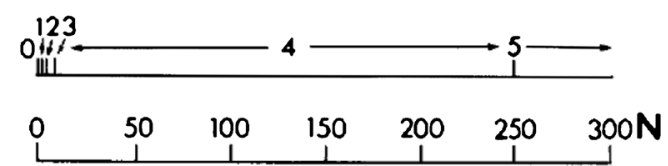

Fig. 1 A comparison between Medical Research Council strength grading and quantitative strength measurements for biceps femoris. Reprinted with permission from van der Ploeg et al. [15]. Copyright 1984 Journal of Neurology 
pulmonary function, and timed motor tasks. With respect to muscle strength, standardized patient positions were defined, and a strain gauge moved around the patient to be orthogonal to specific muscle groups was used to measure isometric strength. The full battery included 9 muscle groups measured bilaterally, plus handgrip measured with a separate grip dynamometer.

Longitudinal studies of patients with ALS demonstrated a number of important findings. First, though careful patient positioning and rigorous evaluator training resulted in reproducible measurements for individual muscles over time, reliability was increased if certain muscle groups were considered together. To be determined which muscle groups could most effectively be combined, Andres et al. [21] performed a factor analysis on strength data from single studies of 176 patients with ALS to determine how different muscle groups were intercorrelated. This analysis showed that muscles from the arms could effectively be combined, as could muscles form the legs. As absolute strength for different muscles can be vastly different, each muscle strength measurement was linearly transformed to a $z$ score, using ALS population means and SDs for every muscle. Muscles in different body regions were then averaged to yield a more global value called a megascore. Declines in megascores for the arms and legs were, in general, very linear over time; however, significant differences were noted both from patient to patient and from one area of the body to another. Decline in leg strength was slightly slower than arm strength in patients with ALS patients [22].

Several studies have directly compared quantitative strength testing with MMT grading. Andres et al. [16] compared progression in patients with ALS using both TQNE and MMT grading, and noted that reproducibility and sensitivity to decline were both strikingly greater using quantitative evaluations. The Great Lakes ALS Consortium [14] evaluated both the effect of averaging strength in multiple muscles and the relative sensitivity to change with MMT testing and TQNE. Not surprisingly, the characteristics of the measure improved with number of muscles evaluated both for TQNE and MMT. However, for any given number of muscles averaged, the characteristics of TQNE were superior to that of MMT grading.

Quantitative isometric strength using the TQNE system has been used in several multicenter ALS trials. In a trial of celecoxib in ALS [23], no significant differences between celecoxib and placebo were found, including for muscle strength. As previously noted, leg strength declined slightly slower than arm strength. In a trial of topiramate in ALS [24], only arm strength was measured. A deleterious effect was found for topiramate on all measures; this effect was not statistically significant for the ALSFRS-R or for vital capacity, but was significant for the arm megascore. The percent difference over time between treatment groups was greatest for the arm megascore compared with other measures. A small, phase II study comparing talampenal $50 \mathrm{mg}$ orally 3 times daily to placebo in 60 patients over 9 months showed a trend toward reduced strength loss in patients treated with talampenal; a subsequent phase III study using MMT testing did not confirm this finding [25].

Both natural history studies and clinical trial data show that quantitative strength testing using the TQNE apparatus provided high-quality, reproducible data on muscle strength. However, in the clinical trial setting, several aspects of testing proved problematic. First, the apparatus was quite large, requiring a full examination room. Many clinical trial sites found that committing a full room for testing that occurred occasionally at most was too great an investment. Second, to test all of the muscles suggested in the original evaluation required that patients assume a variety of positions on a physical therapy table, including fully supine and fully prone. Such position changes were fatiguing for many patients, and the appropriate positioning was not possible for patients who had orthopnea. Thus, as the trial progressed, an increasing number of patients were unable to complete the evaluation. In addition, a trained physical therapist or other clinician was required to perform the test.

To address these issues, the use of a hand-held dynamometer was proposed. Such dynamometers have been in frequent use in a variety of clinical situations, primarily to assess recovery after stroke or injury. In spinal cord-injured patients, use of a hand-held myometer was much more reliable than MMT testing [26]. In the clinic, quantitative strength measurements with a hand-held device has been useful in a variety of neuromuscular diseases $[17,27]$. In general, however, reproducibility was not as good for TQNE in most cases, and issues were raised about variability of both patient and evaluator positioning, as well as the fact that, for strong muscles, the strength of the evaluator might be less than the patient [21]. Another source of variability lay in the fact that some protocols required a "break" in position while performing testing; that is, in order to perform a test successfully, the evaluator must overcome the muscle force exerted by the patient. This requires more evaluator strength than the "make" maneuver, in which the evaluator simply matches the strength exerted by the patient. However, in normal volunteers, there is $<3 \%$ difference between forces measured in the make versus break technique, suggesting that evaluator muscle strength may be less of a source of variability than originally proposed [28].

Despite the abovementioned considerations, quantitative strength testing using a hand-held dynamometer (HHD) has been implemented in a large number of clinical trials in ALS and SMA in recent years. To address issues of evaluator variability, a rigorous training and evaluation program was implemented, with standard patient and evaluator positions mandated and a prespecified level of test-retest performance required. In ALS, trials of lithium, ceftriaxone, dexpramipexole, 
and tirasemtiv have been successfully performed, with reliable data acquired that have characteristics suggesting that measurements of muscle strength with HHD is a sensitive measure of disease progression and therapeutic efficacy [29-32]. For lithium, ceftriaxone, and dexpramipexole, HHD data closely matched other outcomes, including ALSFRS-R and slow vital capacity, and survival. However, in a metaanalysis of 3 small, phase II studies of tirasemtiv, strength testing using HHD showed a statistically significant benefit of active treatment with tirasemtiv (Fig. 2) [33-35]. A subsequent large, phase II study of the same agent showed a significant amelioration of rate of progression of muscle weakness with tirasemtiv [32]. A significant benefit was also seen with slow vital capacity, which is actually another measure of maximal muscle strength. However, a signal was not seen in the ALSFRS-R, suggesting that quantitative tests of muscle strength may be more sensitive than a self-report rating scale.

In order to more fully understand the relationships between quantitative strength measurements using HHD and other measures of ALS progression, as well as how different muscle groups change with respect to each other, the placebo groups of 2 recent, large, phase III ALS trials were analyzed [36]. For both data sets, strength in individual muscles or expressed as megascores declined over time. $\mathrm{CoV}(\mathrm{r})$ was chosen as the best measure to incorporate both variability in rate change and variability of measurement; using this measure, knee extension was the most variable measure. CoV(r) for ALSFRS-R suggested slightly less variability than for strength, but measurements of vital capacity were more variable than either of the other 2 measures. Rates of decline of these 3 measures correlated with each other with Pearson $r$ 's, ranging from 0.71 to 0.40 . Rates of decline in muscle strength were, for the most part, highly correlated from side to side, with correlation coefficients ranging from 0.82 to 0.43 (Fig. 3). While

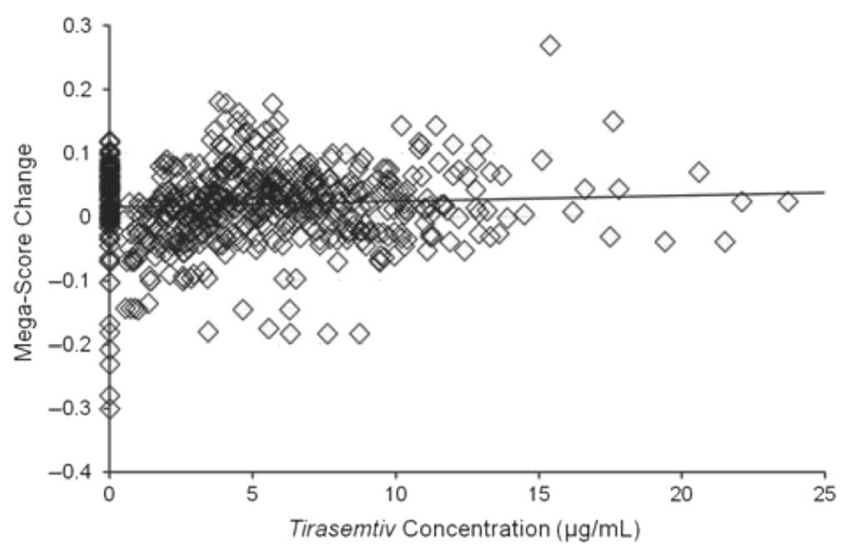

Fig. 2 Quantitative muscle strength measured using hand-held dynamometry as a function of serum concentration of tirasemtiv from a meta-analyis of 3 small, phase II studies. The trend for increased strength as a function of serum concentration is statistically significant $(p<0.002)$. Reprinted with permission from Shefner et al. [34]. Copyright 2013 Amyotrophic lateral sclerosis \& frontotemporal degeneration outliers certainly exist in which side-to-side strength is quite different, the data suggest that, despite the view that ALS is a disease of focal onset and progression, by the time many patients participate in clinical trials, the disease has reached a more disseminated stage with muscle groups declining at similar rates.

While studies of quantitative strength using HHD have provided important insights into the pattern of disease progression in ALS, and HHD has been used frequently in ALS clinical trials, strength measurement has been questioned as an important clinical trial endpoint for several reasons. First, rate of decline in extremity muscle strength does not strongly correlate with survival [37]. The reason for this poor correlation, however, is clear. Death in ALS is almost always due to respiratory failure, an aspect of loss of strength not captured in extremity measurements. Second, power analyses comparing sample sizes required to show meaningful effects in different outcome measures have suggested that the ALSFRS-R has the potential to show a statistically significant difference with fewer patients per group than other measures, including quantitative muscle strength and vital capacity $[36,38]$. These estimates of power are derived primarily from 2 factors: rate of change in the measure over time, and variability across patients. While important factors, neither addresses the question of whether a measure is sensitive to change as a function of a specific therapeutic agent. For example, a recent phase II trial of tirasemtiv, an agent intended to influence muscle strength, showed a robust effect on muscle strength and no effect on ALSFRS-R [32]. Finally, a demonstration of a clinical effect on a functional rating scale provides little insight into what the effect actually is; the ALSFRS-R is a 12-item scale encompassing a range of functions so that an effect on this measure may or may not be clinically meaningful. For all of these reasons, it seems clear that assessment of muscle strength in a disease characterized by progressive weakness should be considered as an important component of clinical trial design.

Quantitative strength measurement using a HHD has also been used in studies of other motor neuron diseases. A small, open-label trial of valproic acid in patients with SMA types III/IV of ages 17 and older showed an increase in strength as measured by HHD in an open-label setting [39]. However, a placebo-controlled trial in adults with SMA also showed no effect, though quantitative strength testing was found to be reliable and reproducible in a trial of valproic acid in SMA [40]; results suggested no benefit but the properties of the strength assessment suggested that it would be a good measure in future trials. Similarly, a trial of type II/III SMA enrolled patients aged 6 to 36 years in a crossover study of growth hormone; HHD was again found to be reliable and reproducible, although the results suggested no effect [41].

Children as young as 5 were enrolled in a natural history study of quantitative strength testing in SMA prior to the onset 
Fig. 3 Side-to-side correlations between 3 muscle groups (elbow flexion, shoulder flexion, ankle dorsiflexion) in both the ceftriaxone and dexpramipexole clinical trials. Reprinted with permission from Shefner et al. [37]. Copyright 2004 Neurology
Ceftriaxone Slopes (z scores)
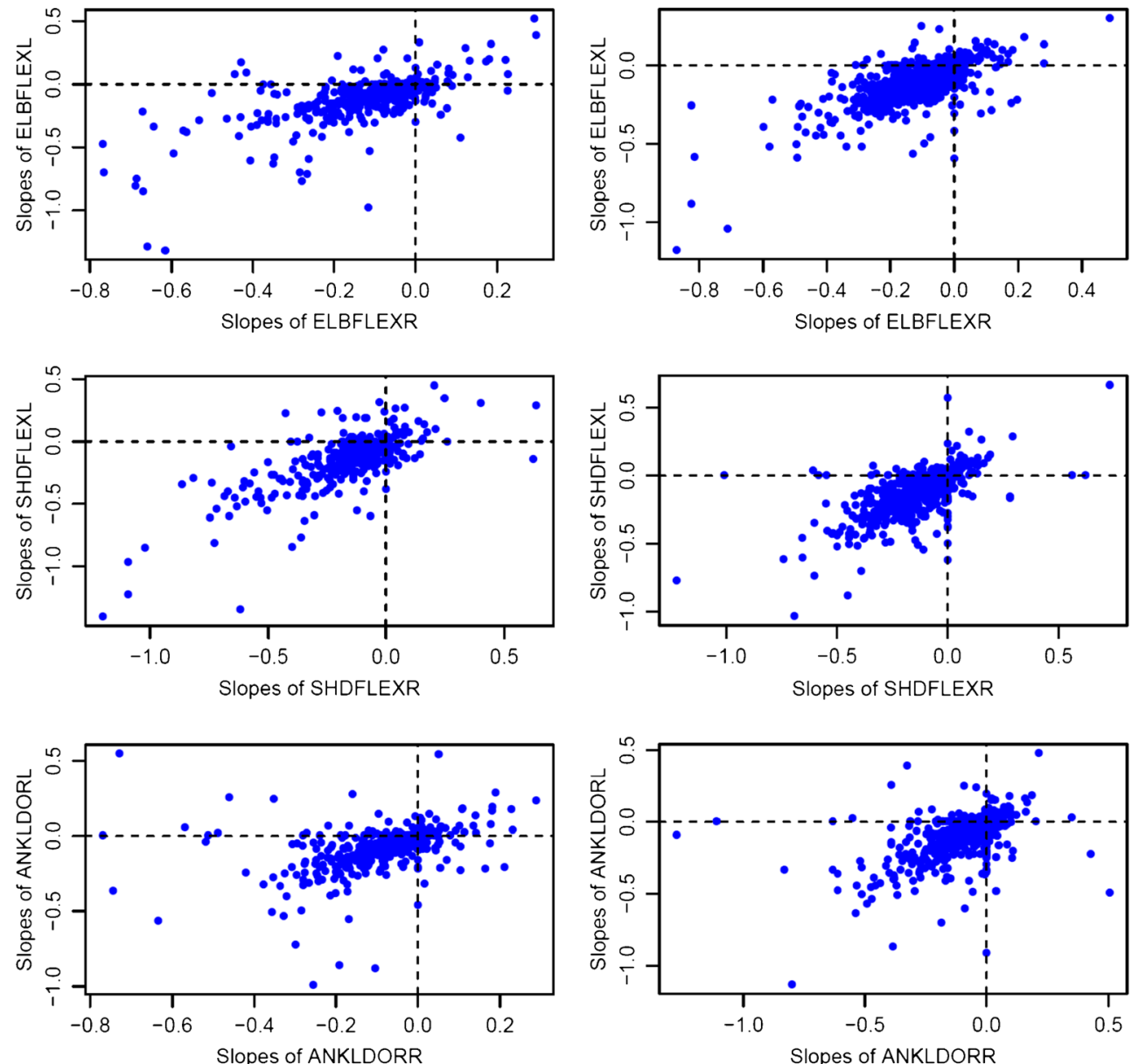

of a clinical trial [42]. Interrater reliability was higher for upper than lower limbs but was quite good for both (intraclass correlation of $0.92-0.98$ for upper limb muscles and $>0.85$ for all lower limb muscles except ankle dorsiflexion). Interrater reliability was also very good with an intraclass correlation of $>0.91$ for all muscles.

Despite the clear advantages of quantitative muscle testing using HHD over MMT strength grading, concern is still expressed over the possibility that, for very strong muscles, the strength of the patient may be greater than the evaluator such that the evaluators strength is being measured rather than that of the patients. To address this issue, Andres et al. [43] have recently developed a modification of the TQNE system, in which patients are seated in a chair and exert effort against an immobile strain gauge rather than an evaluator. Called ATLIS, the apparatus measures 6 muscle groups bilaterally (grip, elbow and knee flexion and extension, and ankle dorsiflexion). In a study of 432 normal patients, ATLIS was highly reproducible, and evaluations could be rapidly obtained without the multiple different subject positions required for
TQNE. Regression equations were established for males and females that described changes in muscle strength with age for the 6 muscle groups tested. Such datasets will be extremely valuable to scale values obtained from diseased patients over a wide range of neuromuscular disorders. Whether the restricted group of muscles reduces the overall quality of a combined muscle measure is yet to be determined.

In summary, strength measures have been used over many years to assess therapeutic benefit in clinical trials for motor neuron diseases. Quantitative measurements have clear advantages over qualitative muscle testing, and a range of techniques are now being incorporated into clinical trials. The use of such measurements have led to increased understanding of common patterns of disease progression in ALS; quantitative strength testing should be incorporated into any trial evaluating an agent intended to either slow motor dysfunction or to cause improvement. Available tools are not perfect; for example, it is currently not possible to distinguish between weakness caused by lesions anywhere in the neuraxis from muscle 
to motor cortex. The ability to determine objectively the source of weakness would be of great value and should be a subject of future research.

Required Author Forms Disclosure forms provided by the authors are available with the online version of this article.

\section{References}

1. Wright W. Muscle training in the treatment of infantile paralysis. Boston Med Surg J 1912;167:567.

2. Council MR. Aids to the examination of the peripheral nervous system. War Memorandum. London: HMSO; 1943.

3. Bensimon G, Lacomblez L, Meininger V. A controlled trial of riluzole in amyotrophic lateral sclerosis. ALS/Riluzole Study Group. N Engl J Med 1994;330:585-591.

4. Lacomblez L, Bensimon G, Leigh PN, Guillet P, Meininger V. Dose-ranging study of riluzole in amyotrophic lateral sclerosis. Amyotrophic Lateral Sclerosis/Riluzole Study Group II. Lancet 1996;347:1425-1431.

5. Groeneveld GJ, van Kan HJ, Lie AHL, Guchelaar HJ, van den Berg LH. An association study of riluzole serum concentration and survival and disease progression in patients with ALS. Clin Pharmacol Ther 2008;83:718-722.

6. Gordon PH, Moore DH, Miller RG, et al. Efficacy of minocycline in patients with amyotrophic lateral sclerosis: a phase III randomised trial. Lancet Neurol 2007;6:1045-1053.

7. Miller R, Bradley W, Cudkowicz M, et al. Phase II/III randomized trial of TCH346 in patients with ALS. Neurology. 2007;69(8):776-84.

8. Chen TH, Chang JG, Yang YH, et al. Randomized, double-blind, placebo-controlled trial of hydroxyurea in spinal muscular atrophy. Neurology 2010;75:2190-2197.

9. Young SD, Montes J, Kramer SS, et al. Six-minute walk test is reliable and valid in spinal muscular atrophy. Muscle Nerve 2016 Mar 25.

10. Werlauff U, Vissing J, Steffensen BF. Change in muscle strength over time in spinal muscular atrophy types II and III. A long-term follow-up study. Neuromuscul Disord 2012;22:1069-1074.

11. Mercuri E, Bertini E, Messina S, et al. Randomized, double-blind, placebo-controlled trial of phenylbutyrate in spinal muscular atrophy. Neurology 2007;68:51-55.

12. Miller RG, Moore DH, Dronsky V, et al. A placebo-controlled trial of gabapentin in spinal muscular atrophy. J Neurol Sci 2001;191:127-131.

13. Querin G, D'Ascenzo C, Peterle E, et al. Pilot trial of clenbuterol in spinal and bulbar muscular atrophy. Neurology 2013;80:2095-2098.

14. Great Lakes ALSSG. A comparison of muscle strength testing techniques in amyotrophic lateral sclerosis. Neurology 2003;61:1503-1507.

15. van der Ploeg RJ, Oosterhuis HJ, Reuvekamp J. Measuring muscle strength. J Neurol 1984;231:200-203.

16. Andres PL, Skerry LM, Thornell B, Portney LG, Finison LJ, Munsat TL. A comparison of three measures of disease progression in ALS. J Neurol Sci 1996;139(Suppl.):64-70.

17. Wiles CM, Karni Y. The measurement of strength in patients with peripheral neuromuscular disorders. J Neurol Neurosurg Psychiatry 1983;46:1006-1013.

18. Andres P, W H, Finison L, Conlon T, Felmus M, Munsat T. Quantitative motor assessment in amyotrophic lateral sclerosis. Neurology 1986;36:937-941
19. Andres PL, English R, Mendoza M, et al. Developing normalized strength scores for neuromuscular research. Muscle Nerve 2012;47:177-182.

20. Andres PL, Finison LJ, Conlon T, Thibodeau LM, Munsat TL. Use of composite scores (megascores) to measure deficit in amyotrophic lateral sclerosis. Neurology 1988;38:405-408.

21. Andres PL, Thibodeau LM, Finison LJ, Munsat TL. Quantitative assessment of neuromuscular deficit in ALS. Neurol Clin 1987;5:125-141.

22. Munsat TL, Andres PL, Finison L, Conlon T, Thibodeau L. The natural history of motoneuron loss in amyotrophic lateral sclerosis. Neurology 1988;38:409-413.

23. Cudkowicz ME, Shefner JM, Schoenfeld DA, et al. Trial of celecoxib in amyotrophic lateral sclerosis. Ann Neurol 2006;60:22-31.

24. Cudkowicz ME, Shefner JM, Schoenfeld DA, et al. A randomized, placebo-controlled trial of topiramate in amyotrophic lateral sclerosis. Neurology 2003;61:456-464.

25. Pascuzzi RM, Shefner J, Chappell AS, et al. A phase II trial of talampanel in subjects with amyotrophic lateral sclerosis. Amyotroph Lateral Scler 2010;11:266-271.

26. Schwartz S, Cohen ME, Herbison GJ, Shah A. Relationship between two measures of upper extremity strength: manual muscle test compared to hand-held myometry. Arch Phys Med Rehabil 1992;73:1063-1068.

27. Wiles CM, Karni Y, Nicklin J. Laboratory testing of muscle function in the management of neuromuscular disease. J Neurol Neurosurg Psychiatry 1990;53:384-387.

28. van der Ploeg RJ, Oosterhuis HJ. The "make/break test" as a diagnostic tool in functional weakness. J Neurol Neurosurg Psychiatry 1991;54:248-251.

29. Aggarwal SP, Zinman L, Simpson E, et al. Safety and efficacy of lithium in combination with riluzole for treatment of amyotrophic lateral sclerosis: a randomised, double-blind, placebo-controlled trial. Lancet Neurol 2010;9:481-488.

30. Cudkowicz ME, Titus S, Kearney M, et al. Safety and efficacy of ceftriaxone for amyotrophic lateral sclerosis: a multi-stage, randomised, double-blind, placebo-controlled trial. Lancet Neurol 2014;13:1083-1091.

31. Cudkowicz ME, van den Berg LH, Shefner JM, et al. Dexpramipexole versus placebo for patients with amyotrophic lateral sclerosis (EMPOWER): a randomised, double-blind, phase 3 trial. Lancet Neurol 2013;12:1059-1067.

32. Shefner JM, Wolff AA, Meng L, , et al. A randomized, placebocontrolled, double-blind phase IIb trial evaluating the safety and efficacy of tirasemtiv in patients with amyotrophic lateral sclerosis. Amyotroph Lateral Scler Frontotemporal Degener 2016;17:426-435.

33. Shefner JM, Watson ML, Meng L, Wolff AA, Neals/Cytokinetics ST. A study to evaluate safety and tolerability of repeated doses of tirasemtiv in patients with amyotrophic lateral sclerosis. Amyotroph Lateral Scler Frontotemporal Degener 2013;14:574-581.

34. Shefner JM, Wolff AA, Meng L. The relationship between tirasemtiv serum concentration and functional outcomes in patients with ALS. Amyotroph Lateral Scler Frontotemporal Degener 2013;14:582-585.

35. Shefner J, Cedarbaum JM, Cudkowicz ME, et al. Safety, tolerability and pharmacodynamics of a skeletal muscle activator in amyotrophic lateral sclerosis. Amyotroph Lateral Scler 2012;13:430-438.

36. Shefner JM, Liu D, Leitner ML, et al. Quantitative strength testing in ALS clinical trials. Neurology 2016;87:617-624.

37. Traynor BJ, Zhang H, Shefner JM, Schoenfeld D, Cudkowicz ME. Functional outcome measures as clinical trial endpoints in ALS. Neurology 2004;63:1933-1935. 
38. Rutkove SB, Caress JB, Cartwright MS, et al. Electrical impedance myography as a biomarker to assess ALS progression. Amyotroph Lateral Scler 2012;13:439-445.

39. Weihl CC, Connolly AM, Pestronk A. Valproate may improve strength and function in patients with type III/IV spinal muscle atrophy. Neurology 2006;67:500-501.

40. Kissel JT, Elsheikh B, King WM, et al. SMA valiant trial: a prospective, double-blind, placebo-controlled trial of valproic acid in ambulatory adults with spinal muscular atrophy. Muscle Nerve 2014:49:187-192
41. Kirschner J, Schorling D, Hauschke D, et al. Somatropin treatment of spinal muscular atrophy: a placebo-controlled, double-blind crossover pilot study. Neuromuscul Disord 2014;24:134-142.

42. Merlini L, Mazzone ES, Solari A, Morandi L. Reliability of handheld dynamometry in spinal muscular atrophy. Muscle Nerve 2002;26:64-70

43. Andres PL, Skerry LM, Munsat TL, et al. Validation of a new strength measurement device for amyotrophic lateral sclerosis clinical trials. Muscle Nerve 2012;45:81-85. 